\title{
DO EMERGENCY SERVICES FOCUS ENOUGH ON PRE-HOSPITAL HYPOTHERMIA MANAGEMENT?
}

\author{
Kobi Ludwin
}

Polish Society of Disaster Medicine, Poland

Disaster Emerg Med J 2017; 2(2): 100-101

Dear Editor,

The importance of hypothermia treatment during and after emergency care has been emphasized and described in countless trials and papers. Its role and weight in the various critical care protocols, both civilian and military, is common knowledge, as well as being advocated by many and practiced, to an extent, by many emergency services [1]. However, its use during prehospital management by different emergency services worldwide, not necessarily using the same disciplines and practices, leaves much to be desired. Moreover, it does not always get the attention it deserves when directing budgets or choosing a solution proven to be properly effective for hypothermia treatment or its prevention in the field.

The loss of body heat may occur through four different processes: radiation, evaporation, conduction and convection [2]. Even though this is common knowledge, most of the hypothermia prevention and treatment devices used by emergency medical services today do not offer a significant solution to all these heat loss mechanisms. The most common device used today, the space blanket, does not present viable hypothermia treatment in most, if not all, prehospital clinical settings. In order to truly prevent trauma patient hypothermia, the caregiver should keep in mind and address all of these heat loss mechanisms during prehospital field treatment.

Hypothermia is relatively common in trauma victims and has been associated with increased mortality, being reported to have occurred in up to two thirds of described patients. It has been concluded that adequate hypothermia prevention measures and immediate thermal protection should be routine and should be initiated as soon as EMS teams arrive at the scene [3].
There are tested and proven devices for the prevention and treatment of prehospital hypothermia: devices that have been recommended and named in tactical combat casualty care [4]. These devices have been adopted and trialled over the years by military combat care givers during casualty trauma management.

A torso model comparison of temperature preservation devices for use in the prehospital environment was demonstrated by Zaza, Flowers, Zidelman, Hodgetts and Haris in 2016. They detetcted a significant difference in temperature between the Blizzard blanket, named in the tactical combat casualty care protocol as a blanket of choice for hypothermia prevention, and the ready heat (active heating), compared to other devices commonly used by emergency services such as the space blanket, bubble wrap and ambulance blanket [5].

Blizzard Protection Systems (Wales) recently released the Blizzard EMS, a hypothermia prevention blanket designed for the civilian arena. Its design, size and thermal protection has been adjusted to better suit the needs of civilian emergency medical services [6].

Untreated hypothermia of the trauma casualty is known to be part of the trauma triad of death, alongside coagulopathy and acidosis [7]. This, and being exposed to all of the above, should lead emergency services, those which are yet to put more emphasize on pre-hospital hypothermia management and the methods they use to do so, to choose effective hypothermia treatment and prevention devices. With the advance in technology, ease of use and cost effectiveness of real, proven and efficient prehospital hypothermia treatment devices, the author hopes that more emergency services around the globe will adopt proper trauma hypothermia management and thereby, inevitably, save more lives. 
Conflict of interest: None declared.

\section{REFERENCES}

1. Navarro KW. Hypothermia and trauma: A deadly combination. https:// www.ems1.com/ems-products/Bleeding-Control/articles/1189729-Hypothermia-and-trauma-A-deadly-combination/ (01.12.2011).

2. http://www.webmd.com/first-aid/tc/cold-exposure-ways-the-bodyloses-heat-topic-overview.

3. Lapostolle F, Couvreur J, Koch FX, et al. Hypothermia in trauma victims at first arrival of ambulance personnel: an observational study with as- sessment of risk factors. Scand J Trauma Resusc Emerg Med. 2017; 25(1): 43, doi: 10.1186/s13049-017-0349-1, indexed in Pubmed: 28438222.

4. http://www.trngcmd.marines.mil/Portals/207/Docs/FMTBE/Student\%20Materials/TCCCGuidelines160603.pdf.

5. Zasa $\mathrm{M}$, Flowers $\mathrm{N}$, Zideman $\mathrm{D}$, et al. A torso model comparison of temperature preservation devices for use in the prehospital environment. Emerg Med J. 2016; 33(6): 418-422, doi: 10.1136/ emermed-2015-204769, indexed in Pubmed: 26838037.

6. http://www.blizzardsurvival.com/article.php/59/blizzard-ems-blanket.

7. Gerecht R. The lethal triad. Hypothermia, acidosis \& coagulopathy create a deadly cycle for trauma patients. JEMS. 2014; 39(4): 56-60. 